\title{
Radiocirurgia no tratamento das metástases cerebrais
}

\author{
Marcos Vinícius Calfat Maldaun', Paulo Henrique Pires de Aguiar², Helder José \\ Lessa Zambelli ${ }^{3}$, Manoel Jacobsen Teixeira ${ }^{4}$, Raymond Sawaya ${ }^{5}$, Frederic Lang ${ }^{5}$
}

\section{RESUMO}

A radiocirurgia vem ganhando espaço no meio neurocirúrgico como opção de tratamento para metástases cerebrais. Baixo custo, altas taxas de controle local da doença, baixos índices de complicações relatados na literatura, possibilidade de tratar lesões não cirúrgicas, controle da doença sem as complicações tardias da radioterapia e natureza não invasiva do tratamento ratificam a radiocirurgia como modalidade eficaz no controle do câncer do sistema nervoso central. Nesta revisão, conclui-se que melhor detalhamento das complicações, especialmente as tardias, e estudo randomizado comparando-a com cirurgia seriam de grande interesse.

\section{PALAVRAS-CHAVE}

Metástase cerebral. Radiocirurgia.

\begin{abstract}
Sterotactic radiosurgery for brain metastases

Radiosurgery has become more useful in the treatment of brain metastases. Low costs, high rates of local tumor control, low rates of complications, possibility to treat non surgical cases, absence of brain damage as due to radiotherapy and the non invasive treatment concept supports the benefits of radiosurgical modality. However, this review conclude that further studies based on longer follow up periods to detect late complications as well as prospective studies comparing surgical and radiosurgical results will be of a great interest to clarified questions still in need.
\end{abstract}

\section{KEY WORDS}

Brain metastases. Stereotactic radiosurgery.

\section{Introdução}

A radiocirurgia estereotáxica (RCE) foi desenvolvida pelo neurocirurgião sueco Lars Leksell em 1951. Leksell desenvolveu essa técnica capaz de destruir uma lesão-alvo estipulada com uma dose única e alta de radiação por meio de estreitos colimadores. A técnica oferece rápida queda de dose nas margens da lesão, permitindo segura proteção do cérebro e dos tecidos adjacentes. Em 1968, desenvolveu-se na Suécia a unidade Gamma. No decorrer das décadas subseqüentes, principalmente na de 1980, na Europa, com a melhora das técnicas diagnósticas e com processadores de imagem tridimensionais, o uso potencial da RCE alastrou-se para o tratamento de outras doenças do sistema nervoso central (SNC), como malformações arteriovenosas, doença de Cushing, neurinomas de acústico, doença de Parkinson, bem como para o tratamento da dor.

O sistema radiocirúrgico desenvolvido por Leksell tornou-se conhecido como Gamma Knife (GK). Outros sistemas foram desenvolvidos, destacando-se o acelerador linear (LINAC). A RCE no tratamento de metástases cerebrais foi iniciada na década de 1980, por Sturm e cols. ${ }^{64}$, que, com LINAC, administraram

1 Hospital Santa Paula, Hospital Sírio-Libanês, Hospital Estadual Sumaré - Universidade Estadual de Campinas (Unicamp).

2 Hospital Santa Paula, Hospital Sírio-Libanês, Divisão de Clínica Neurocirúrgica do Hospital das Clínicas da Faculdade de Medicina da Universidade de São Paulo (FMUSP).

3 Hospital Estadual Sumaré - Unicamp.

4 Hospital 9 de Julho, Hospital Sírio-Libanês, Divisão de Neurocirurgia Funcional do Instituto de Psiquiatria do Hospital de Clínicas da FMUSP.

5 M.D. Anderson Cancer Center Hospital, Houston, Texas, Estados Unidos. 
altas doses (entre 2.000 e 3.000 cGy) em 12 lesões inoperáveis e rádio-resistentes, obtendo redução do volume e do edema perilesional e melhora dos sintomas causados pelas lesões. Logo após, Loeffler e cols. ${ }^{34}$ confirmaram a efetividade da RCE no tratamento das metástases cerebrais recorrentes. Desde então, diversas publicações têm mostrado a capacidade da RCE no tratamento das metástases cerebrais, especialmente com otimização das doses, identificação dos grupos de risco de complicação e fatores prognósticos, com maiores períodos de acompanhamento pós-tratamento. Atualmente, nos centros americanos de unidade Gamma, as metástases cerebrais somam mais de 1/3 dos $\operatorname{casos}^{62}$, mas cada serviço apresenta sua própria característica epidemiológica.

\section{Radiobiologia}

Após colocação do aro estereotáxico, que se encaixa dentro de uma câmara de cobalto (GK), ou ao LINAC com tecnologia rotatória, são traçadas coordenadas tridimensionais estereotáxicas, definindo o ponto central ou isocentro, que consiste no centro da lesão-alvo. O sistema serve de fonte de partículas, por meio de vários canais, concentrando doses radioativas no alvo em questão; pelos colimadores secundários (de vários tamanhos) são estabelecidos arcos ao redor do alvo. A dose acumula-se no centro, caindo rapidamente nas bordas da lesão mapeada. O número de arcos utilizados depende do formato da lesão. A prescrição da dose radiocirúrgica vem sendo otimizada empiricamente de acordo com a radiobiologia e repercussões clínicas radioterapêuticas, tentando estabelecer um equilíbrio entre efetividade e tolerabilidade.

A radiocirurgia visa à destruição precisa e completa de um alvo escolhido contendo tecido normal e/ou patológico, sem significativo dano pela radiação, seja concomitante ou tardio, de tecidos adjacentes ${ }^{29}$. O radiobiologista Van der Kogel acredita que o conceito de radiobiologia com RCE (dose única) é diferente da irradiação fracionada ${ }^{29,72}$. A diferença entre RCE e radioterapia é o tamanho do volume a ser tratado. Um exemplo disso é que como a radioterapia trata uma grande área, geralmente envolvendo extenso volume de tecido normal, doses menores devem ser aplicadas. Assim, muitas lesões tidas como rádio-resistentes tornam-se responsivas às doses maiores e únicas da $\mathrm{RCE}^{4,7-}$ $9,11,13,15,18,20,23,26,27,35,38,40,42,43,45,48,50,56,61,63,70$. O alvo estabelecido e preciso é a essência dos efeitos radiobiológicos da RCE. Ainda seus efeitos são estudados, e uma série de questões permanece sem resposta, como efeitos em diversas doenças neurológicas, áreas epileptogênicas, interação farmacológica e a diversidade de resposta para casos de pacientes com a mesma patologia.

Preconiza-se que a RCE cause vasculopatia proliferativa em uma malformação arteriovenosa, começando com lesão endotelial secundária às altas doses de radiação, tornando os vasos hialinizados e mais finos, levando à oclusão do lúmen. Como as metástases cerebrais são tumores caracterizados por sobreviver em situações hipóxicas, pressupõe-se que a alta dose radioativa ministrada cause combinação de lesão citotóxica com degeneração celular e efeitos vasculares tardios. A maioria dos estudos anatomopatológicos de metástases cerebrais tratadas com RCE mostra áreas de necrose coagulativa e células tumorais não viáveis ${ }^{25,29}$. Szeifert e cols. ${ }^{67}$ realizaram análise imunoistoquímica em cinco casos de metástases cerebrais tratadas com RCE, ratificando que células endoteliais vasculares são o principal alvo da alta e única dose da RCE. Os mesmos autores também evidenciaram ${ }^{68}$ resposta imune celular contra células tumorais, provavelmente estimulada pela energia ionizante da radiação focal. Os fatores de necrose tumoral e outros fatores liberados com o tratamento e suas relações com as metaloproteinases e outras proteínas na matriz extracelular devem causar o edema peritumoral e o grau variado de captação anelar, mas tais efeitos e relações da microbiologia tumoral e radiobiologia ainda estão sob estudo.

\section{Radiocirurgia no tratamento das metástases cerebrais}

Desde o início da utilização da RCE para o tratamento das metástases cerebrais, centenas de publicações relatam sua alta taxa de efetividade e baixas taxas de complicações. A tabela 1 mostra os resultados obtidos em grandes casuísticas, principalmente na década de 1990. Em geral, a taxa de controle local da lesão varia de $85 \%$ a $95 \%$ dos casos. Huber e cols. ${ }^{24}$ apresentam taxa de controle local de $91 \%$, em dois anos de acompanhamento, de 87 metástases cerebrais tratadas com RCE. Ulm e cols. ${ }^{71}$ relataram $75 \%$ de controle em sua série com 383 pacientes. Sansur e cols. ${ }^{52}$ apresentaram taxas de controle local de $82 \%$ em 173 pacientes tratados. Simonova e cols. ${ }^{61}$ trataram com RCE 237 pacientes e obtiveram controle local em $94,9 \%$ dos casos. Li e cols. ${ }^{31}$ compararam resultados obtidos com a RCE à radioterapia convencional e à associação de ambas, obtendo melhores resultados com RCE e RCE associada à radioterapia do que com radioterapia apenas. Resultados similares foram encontrados por Kocher e cols. ${ }^{28}$ comparando RCE com radioterapia. Gerosa e cols. ${ }^{13}$ estudaram retrospectivamente 804 pacientes com mais de 1.300 metástases cerebrais e observaram controle local em $94 \%$ dos casos. 
Tabela 1.

Publicações recentes sobre $R C E$

\begin{tabular}{|c|c|c|c|c|c|}
\hline Autor/ano & № de casos & Histologia & $\begin{array}{l}\text { Controle local (\%) e } \\
\text { sobrevida média }\end{array}$ & $\begin{array}{l}\text { Dose média } \\
\text { (Gy) }\end{array}$ & Volume $\left(\mathrm{cm}^{3}\right)$ \\
\hline Matsuo, 1999 & 162 & Variada & $\begin{array}{l}79,6 \% \\
11 \text { meses }\end{array}$ & 19,5 & 4,49 \\
\hline Shaw, 2000 & 168 & Variada & $\begin{array}{l}60 \% \\
7,5 \text { meses }\end{array}$ & 21 & 8,2 \\
\hline Maor, 2000 & 84 & Variada & $\begin{array}{l}77 \% \\
7 \text { meses }\end{array}$ & 19 & 3,59 \\
\hline Simonova, 2000 & 237 & Variada & $\begin{array}{l}94,9 \% \\
10 \text { meses }\end{array}$ & 21 & 7,5 \\
\hline Kim, 2000 & 121 & Variada & $\begin{array}{l}76 \% \\
12,2 \text { meses }\end{array}$ & 15,4 & 2,1 \\
\hline Brown, 2002 & 83 & "rádio-resistentes" & $\begin{array}{l}88 \% \\
14,2 \text { meses }\end{array}$ & 18 & $20 \mathrm{~mm}^{*}$ \\
\hline Hernandez, 2002 & 92 & $\begin{array}{l}\text { Células } \\
\text { renais }\end{array}$ & 7 meses & 16,8 & 4,7 \\
\hline Serizawa, 2002 & 245 & Adenocarcinoma pulmonar & $\begin{array}{l}94,5 \% \\
9,1 \text { meses }\end{array}$ & 18 & 2,7 \\
\hline Gerosa, 2002 & 1.307 & Variada & $\begin{array}{l}93 \% \\
13,5 \text { meses }\end{array}$ & 20,6 & 4,8 \\
\hline Noel, 2003 & 145 & Adenocarcinoma pulmonar & $\begin{array}{l}91 \% \\
9 \text { meses }\end{array}$ & 18 & $\mathrm{NR}^{* *}$ \\
\hline Gerosa, 2005 & 836 & Adenocarcinoma pulmonar & $\begin{array}{l}94 \% \\
14,5 \text { meses }\end{array}$ & 21,4 & 6,2 \\
\hline Chang, 2005 & 264 & "rádio-resistentes" & 7,5 meses $* * *$ & 18 & 1,6 \\
\hline Shomas, 2005 & 126 & Variada & $\begin{array}{l}88,6 \% \\
8,8 \text { meses }\end{array}$ & 18 & 3 \\
\hline
\end{tabular}

*Maior diâmetro da lesão; ** NR: não relatado; ***Varia com histologia, sendo pior para melanomas e sarcomas.

"Rádio-resistentes": referente a melanomas, células renais e sarcomas.

Em uma série com 424 metástases cerebrais tratadas com RCE, Pan e cols. ${ }^{46}$ relataram controle em $84,4 \%$ das metástases cerebrais de adenocarcinoma de pulmão, ao passo que Gerosa e cols. ${ }^{12}$ atingiram taxas de $94 \%$ para essas lesões. Serizawa e cols. ${ }^{33}$ compararam os resultados de metástases de carcinomas pulmonares de pequenas células e de não pequenas células, com resultados acima de $90 \%$ de controle em ambos. Bindal e cols. ${ }^{5}$ relataram que, em 6 meses de acompanhamento, as metástases tratadas com RCE foram consideradas sem progressão em $86 \%$, ao passo que tal valor cai para $69 \%$ após 1 ano. Já Brown e cols. ${ }^{7}$ evidenciaram taxas de controle de $88 \%$ das metástases chamadas "rádioresistentes". Resultados também satisfatórios foram apresentados por Feuvrete cols. ${ }^{30}$, Gerosa e cols. ${ }^{42}$ e Koc e cols..$^{27}$ para tais lesões. Herfarth e cols. ${ }^{18}$ analisaram pacientes com 122 metástases cerebrais de melanomas, relatando controle local em $82 \%$ dos casos. Já Kim e cols. ${ }^{22}$ mostraram resultados piores para metástases de adenocarcinomas de células renais, ao passo que Chang e cols. ${ }^{8}$ ofereceram resultados de sobrevida melhor para metástases de adenocarcinoma de células renais comparadas com melanoma e sarcoma. Entre 146 metástases cerebrais de câncer de células renais, Sheehan e cols. ${ }^{56}$ mostraram apenas $4 \%$ de crescimento das lesões após tratamento com radiocirurgia estereotáxica. Valor similar foi apresentado por Noel e cols. ${ }^{43}$ em 65 dessas lesões tratadas. As taxas de controle são 
menores quando a RCE é aplicada para recorrência do tumor $^{22}$. Hasegawa e cols. ${ }^{17}$ mostraram que $16,3 \%$ das metástases cerebrais de câncer de gastrointestinal progrediram com o tratamento. Muacevic e cols. ${ }^{39}$, em sua série de 620 metástases de câncer mama tratadas com RCE, referiram taxas de controle local em 94\%.

Publicações recentes têm mostrado efetividade satisfatória no tratamento de metástases cerebrais múltiplas com RCE ${ }^{18,19,32,38,45,49,66,69,75}$. Não houve diferença estatística entre metástases múltiplas cerebrais tratadas com RCE e com RCE associada à radioterapia no estudo feito por Jawahar e cols. ${ }^{60}$, bem como no estudo feito por Hasegawa e cols. ${ }^{16}$.

Assim, como nas séries cirúrgicas, dependendo dos fatores prognósticos temos resultados variados de sobrevida e controle local, bem como a toxicidade de acordo com dose aplicada e ao tamanho das lesões. Pacientes selecionados com recursive partitioning analysis (RPA) I ou II, ou score index for radiosurgery (SIR) entre 8 e 10, apresentam melhores resultados ${ }^{74}$. Goodman e cols..$^{26}$ apresentaram resultados diferentes de acordo com grau de captação de contraste da lesão, com controle melhor para metástases que apresentavam captação homogênea de contraste. Shaw e cols. ${ }^{55}$ colocam como seguras e efetivas as doses de $24 \mathrm{~Gy}, 18 \mathrm{~Gy}$ e $15 \mathrm{~Gy}$, respectivamente, para tumores menores que $20 \mathrm{~mm}$, entre $21 \mathrm{~mm}$ e $30 \mathrm{~mm}$ e entre $31 \mathrm{~mm}$ e $40 \mathrm{~mm}$ de diâmetro. Matsuo e cols..$^{35}$ preconizam a utilização de 25 Gy para lesões menores que $10 \mathrm{~mm}$ e 20 Gy para lesões maiores, com diminuição da dose quando a metástase estiver próxima a áreas de "alto risco". Shehata e cols. ${ }^{58}$ não observaram resultado melhor no controle da doença com doses maiores que $20 \mathrm{~Gy}$, e estas foram associadas a maior taxa de complicações. Schomas e cols. ${ }^{54}$ e Sheehan e cols. ${ }^{57}$ evidenciam que a mínima dose-alvo é fator preditivo de efetividade, bem como o tamanho tumoral, assim como Noel e cols. ${ }^{41}$ e Shuto e cols. ${ }^{60}$. Assim, metástases no tronco cerebral tratadas com dose $<15$ Gy têm taxa de controle inferior (em torno de $77,4 \%$ ).

Alguns trabalhos retrospectivos compararam a efetividade da RCE com os resultados cirúrgicos. Bindal e cols. ${ }^{5}$ compararam 31 pacientes tratados com RCE a 62 pacientes submetidos à ressecção cirúrgica da metástase e evidenciaram melhor sobrevida e controle local das lesões nos casos cirúrgicos, assim como Shinoura e cols. ${ }^{10}$ em 98 lesões estudadas. Já em um estudo com 133 pacientes, Schoggl e cols. ${ }^{53}$ concluíram que ambas são modalidades similares de tratamento. Mesma conclusão é a de outros autores em seus estudos retrospectivos ${ }^{4,23,47}$. Em um estudo multinstitucional, Aucher e cols. ${ }^{2}$ compararam 122 pacientes e também obtiveram resultados similares entre as duas modalidades. O estudo de Cho e cols. ${ }^{10}$, que analisou 225 pacientes tratados com radioterapia, $\mathrm{RCE}$ associada a radioterapia e cirurgia associada a radioterapia, demonstrou que tanto o grupo cirúrgico como o da RCE apresentaram melhor sobrevida que o grupo da radioterapia apenas.

Siomin e cols.$^{68}$ demonstraram menor taxa de carcinomatose meníngea entre pacientes com metástases na fossa posterior tratadas com RCE do que com cirurgia. Em estudos de custo-benefício comparando RCE e cirurgia, Mehta e cols. ${ }^{36} \mathrm{e}$ Wellis e cols. ${ }^{73}$ apresentaram efetividade similar, mas custo superior de procedimento e de período pós-tratamento para pacientes cirúrgicos devido ao baixo tempo de internação e recuperação dos pacientes tratados com RCE. Não há, ainda, trabalhos publicados com estudos prospectivos e randomizados comparando RCE e cirurgia.

\section{Complicações do tratamento}

Apesar das centenas de publicações sobre RCE no tratamento das metástases cerebrais, faltam estudos detalhados sobre as complicações dessa modalidade terapêutica. A literatura limita-se a descrições sucintas, com pouco tempo de seguimento dos casos, apenas avaliando a efetividade do tratamento, sem especificações das complicações, dependência ao corticóide ou relação do tratamento com lesões nas (ou próximas às) áreas eloqüentes. Como já foi dito, a RCE obtém, em média, uma taxa de falha do tratamento, com recidiva local, de $10 \%$ (variando entre $3 \%$ e $30 \%$ ), porém a maioria dos estudos tem tempo de acompanhamento pós-tratamento pequeno.

Define-se controle da lesão como a manutenção ou a diminuição do tamanho da metástase. Contudo, o paciente pode apresentar manutenção do tamanho da lesão com piora clínica devido a edema, necrose ou fatores de liberação tumoral. Além disso, apenas recentemente ficaram estabelecidas as relações de dose, volume da lesão e localização da lesão com efetividade e neurotoxicidade.

Outro fator interessante é como diferenciar radionecrose com repercussões clínicas de necrose decorrente do tratamento, e ainda a potencialização ou não pela radioterapia pré ou pós-tratamento. Obviamente, quanto maior o volume, maior a chance de complicações e necrose. Conforme constatado por Huber e cols. ${ }^{24}$, pode ocorrer aumento transitório do volume da lesão póstratamento em até $19 \%$ dos casos, sem necessariamente classificar tais casos como falha terapêutica. Por fim, um assunto praticamente ignorado pelos estudos é se a dependência ao corticóide no pós-tratamento deve ou não ser considerada um quesito agravante para o 
paciente. Shehata e cols. ${ }^{58}$ demonstraram que doses superiores a 20 Gy são significativamente associadas a neurotoxicidade com grau 3 ou 4 utilizando a radiation therapy oncology group scale (escala RTOG).

Petrovich e cols. ${ }^{49}$, entre mais de 1.300 lesões tratadas, relataram que em 20 casos $(4,7 \%)$ houve necessidade de craniotomia para remoção de foco necrótico. Em um estudo retrospectivo com 200 casos, Kondziolka ${ }^{30}$ comparou alguns efeitos colaterais da RCE com os da radioterapia, observou que algum tipo de manifestação ocorreu em $34 \%$ e $63 \%$ respectivamente e concluiu que apenas 5\% dos pacientes tratados com RCE se queixaram de fadiga, e $76 \%$ relataram satisfação com o tratamento. Já $\mathrm{O}^{\prime} \mathrm{Neill}^{44}$ não teve complicações em sua série. Liu e cols. ${ }^{33}$ mostraram que $26,7 \%$ dos casos tiveram complicações com o tratamento. Radionecrose e hemorragia pós-tratamento ocorreram, respectivamente, em 12,9\% e 9,7\% no estudo de Bindal e cols 5 . Já Chin e cols. ${ }^{10}$ encontraram 2,2\% de complicações agudas da RCE entre 835 casos tratados, ao passo que Simonova e cols. ${ }^{61}$ constataram toxicidade aguda em $10 \%$ dos casos. Em um estudo enfatizando a avaliação neurocognitiva dos pacientes, Regine e cols. ${ }^{51}$ descreveram que os pacientes com metástases cerebrais são passíveis de realizar bateria de testes neuropsicológicos, sendo estes dificultados principalmente pela piora na performance e não por fatores institucionais. Em uma revisão feita em 2004, Pollock ${ }^{37}$ encontrou quatro casos de tumores induzidos pela RCE, diferentemente de uma incidência maior nos casos tratados com radioterapia. Thomsen e cols. ${ }^{72}$ relataram caso de transformação sarcomatosa de um neurinoma tratado com RCE. Yu e cols. ${ }^{76}$ relataram casos de glioblastoma após tratamento de meningeoma com a RCE.

A grande dificuldade de se concluir uma indução maligna após RCE é a ausência do diagnóstico histopatológico pré-tratamento com $\mathrm{RCE}^{3}$. Suzuki e cols. ${ }^{65}$ identificaram sangramento intratumoral em 7,4\% das metástases antes da RCE e, em 18,5\%, após a RCE, sendo metade dos casos no primeiro mês após o tratamento. Tais sangramentos ocorreram principalmente em tumores maiores e com altas doses no tratamento ( 20 a 25 Gy). Em uma grande revisão da literatura, Boyd e cols. ${ }^{6}$ enfatizam que a dependência ao corticóide e o edema pós-tratamento não são considerados problemas comuns. Shaw e cols. ${ }^{55}$ mostraram que $18 \%$ a $25 \%$ dos casos tratados na vigência de corticoterapia descontinuam o uso desta no primeiro ano, que $31 \%$ a $45 \%$ de casos sem usos de esteróides permanecem sem o mesmo após o tratamento e que $10 \%$ necessitaram iniciar o uso após o tratamento. Os mesmos autores, em outra publicação, constatam que quanto maior o tamanho da lesão tratada e maior a dose aplicada, maiores as incidências de toxicidade graus 3,4 e 5 (tabela 2).
Não há, ainda, estudo detalhado que avalie as diferentes complicações correlacionando proximidade da lesão com áreas eloqüentes, como centro da fala, área motora, área visual e tronco cerebral, bem como a dependência ao corticóide correlacionada a essas específicas regiões funcionais cerebrais. Além disso, não podemos afirmar que a RCE é a modalidade de escolha no tratamento das metástases cerebrais, como vários autores preconizam, visto que tem resultados semelhantes aos do tratamento cirúrgico, mas não há estudo randomizado comparando adequadamente ambas as formas terapêuticas.

\begin{tabular}{|c|c|c|c|}
\hline \multicolumn{4}{|c|}{$\begin{array}{c}\text { Tabela } 2 . \\
\text { Incidência de toxicidade graus } 3,4 \text { e } 5 \\
\text { pelo tamanho tumoral e pela dose }\end{array}$} \\
\hline $\begin{array}{l}\text { Tamanho da } \\
\text { lesão (mm) }\end{array}$ & Dose (Gy) & № de pacientes & $\begin{array}{l}\text { Pacientes } \\
\text { com toxicidade (\%) }\end{array}$ \\
\hline \multirow[t]{3}{*}{$\leq 20$} & 18 & 12 & 8 \\
\hline & 21 & 18 & 11 \\
\hline & 24 & 10 & 10 \\
\hline \multirow[t]{4}{*}{$21-30$} & 15 & 15 & 13 \\
\hline & 18 & 15 & 20 \\
\hline & 21 & 13 & 38 \\
\hline & 24 & 12 & 58 \\
\hline \multirow[t]{3}{*}{$31-40$} & 12 & 21 & 10 \\
\hline & 15 & 22 & 14 \\
\hline & 18 & 18 & 50 \\
\hline
\end{tabular}

Adaptado de Shaw et al..$^{55}$

\section{Referências}

1. AMENDOLA BE, WOLF A, COY S, AMENDOLA MA: Radiosugery as palliation for brain metastases: a retrospective review of 72 patients harboring multiple lesions at presentation. J Neurosurg 97(5 Suppl):511-4, 2002.

2. AUCHER RM, LAMOND JP, ALEXANDER E: A multiinstitutional outcome and prognostic factor analysis of radiosurgery for resectable single brain metastases. Int J Radiat Oncol Biol Phys 35: 27-35, 1996.

3. BANCE M, GUHA A: Radiation-induced malignant tumors after stereotactic radiosurgery. Otology \& Neurology 22:1245, 2001.

4. BARKER FG 2 ${ }^{\text {nd. }}$ : Surgical and radiosurgical management of brain metastases. Surg Clin North Am 85: 329-45, 2005.

5. BINDAL AK, BINDAL RK, HESS KR, SHIU A, HASSENBUSCH SJ, SHI WM et al.: Surgery versus radiosurgery in the treatment of brain metastases. J Neurosurg 84:74854, 1996. 
6. BOYD TS, MEHTA MP: Stereotatic radiosurgery for brain metastases. Oncology (Huntingt) 13:1397-409, 1999.

7. BROWN PD, BROWN CA, POLLOCK BE, GORMAN DA, FOOTE RL: Stereotactic radiosurgery for patients with "radioresistent" brain metastases. Neurosurgery 51:656-67, 2002.

8. CHANG EL, SELEK U, HASSENBUSCH SJ $3^{\text {rd }}$, MAOR MH, ALLEN PK, MAHAJAN A et al.: Outcome variation among "radioresistant" brain metastases treated with stereotactic radiosurgery. Neurosurgery 56:936-45, 2005.

9. CHIARION-SILENI V, MURR R, PIGOZZO J, SARTI S, TOMASSI O, ROMANINI A: Brain metástases from malignant melanoma. Forum (Genova) 13:170-82, 2003.

10. $\mathrm{CHO} \mathrm{KH}, \mathrm{HALL}$ WA, LEE AK: Stereotactic radiosurgery for patients with single brain metastases. J Radiosurg 1:79-85, 1998.

11. D'ANDREA G, ROPERTO R, DINIA L, CAROLI E, SALVATI M, FERRANTE L: Solitary cerebral metastases from ovarian epithelial carcinoma: 11 cases. Neurosurg Ver 28:120-3, 2005

12. GEROSA M, NICOLATO A, FORONI R, TOMAZZOLI L, BRICOLOA: Analysis of long-term outcomes and prognostic factors in patients with non-small cell lung cancer brain metastases treated by gamma knife radiosurgery. J Neurosurg 102 Suppl:75-80, 2005

13. GEROSA M, NICOLATO A, FORONI R, ZANOTTI B, TOMAZZOLI L, MISCUSI M et al.: Gamma knife radiosurgery for brain metastases: a primary therapeutic option. J Neurosurg 97(5 Suppl):515-24, 2002.

14. GEROSA M, NICOLATO A, FORONI R: The role of gamma knife radiosurgery in the treatment of primary and metastatic brain tumors. Curr Opin Oncol 15:188-96, 2003.

15. GIEN LT, KWON JS, D'SOUZA DP, RADWAN JS, HAMMOUD JA, SUGIMOTO AK et al.: Brain metastases from endometrial carcinoma: a retrospective study. Gynecol Oncol 93:524-8, 2004.

16. HASEGAWA T, KONDZIOLKA D, FLICKINGER JC, GERMANWALA A, LUNSFORD LD: Brain metastases treated with radiosugery alone: an alternative to whole brain radiotherapy? Neurosugery 52:1318-26, 2003.

17. HASEGAWA T, KONDZIOLKA D, FLICKINGER JC, LUNSFORD LD: Sterotactic radiosurgery for brain metastases from gastrointestinal tract cancer. Surg Neurol 60:506-14, 2003

18. HERFARTH KK, IZWEKOWA O, THILMANN C, PIRZKALL A, DELORME S, HOFMANN U et al.: Linac-based radiosurgery of cerebral melanoma metastases. Analysis of 122 metastases treated in 64 patients. Strahlenther Onkol 179:366-71, 2003.

19. HILLARD VH, SHIH LL, CHIN S, MOORTHY CR, BENZIL DL: Safety of multiple stereotactic radiosurgery treatments for multiple brain lesions. Neurooncol 63:271-8, 2003.

20. HLATKY R, SUKI D, SAWAYA R: Carcinoid metastases to the brain. Cancer 101:2605-13, 2004.

21. HODGSON DC, GOUMNEROVALC, LOEFFLER JS, DUTTON S, BLACK P, ALEXANDER $3^{\text {rd }} E$ et al.: Radiosurgery in the management of pediatric brain tumors. Int $\mathrm{J}$ Radiation Oncology Biol Phys 50:929-35, 2001.

22. HOFFMAN R, SNEED PK, MCDERMOTT MW, LAMBORN KR, PARK WM, LARSON DA: Radiosurgery for brain metastases from primary lung carcinoma. Cancer J 7:121-31, 2001.

23. HOSHI S, JOKURA H, NAKAMURA H, SHINTAKU I, OHYAMA C, SATOH M et al.: Gamma-knife radiosurgery for brain metastasis of renal cell carcinoma: results in 42 cases. Int J Urol 9:618-25, 2002.

24. HUBER PE, HAWIGHORST H, FUSS M, KAICK GV, WANNENMACHER MF et al.: Transient enlargement of contrast uptake on MRI after linear accelerator (LINAC) stereotactic radiosurgery for brain metastases. Int J Radiation Oncology Biol Phys 49:1339-49, 2001.

25. KAMADA K, MASTUO T, TANI M, IZUMO T, SUKUZI Y, OKIMOTO $T$ et al.: Effects of sterotactic radiosurgery on metastatic brain tumors of various histopathologies. Neuropathol 21:307-14, 2001.

26. KIM DG, CHUNG HT, GWAK HS, PAEK SH, JUNG HW, HAN DH: Gamma knife radiosurgery for brain metastases: prognostic factors for survival and local control. J Neurosurg (Suppl 3) 93:23-29, 2000.

27. KOC M, MCGREGOR J, GRECULA J, BAUER CJ, GUPTA N, GAHBAUER RA: Gamma knife radiosurgery for intracranial metastatic melanoma: an analysis of survival and prognostic factors. J Neurooncol 71:307-13, 2005.

28. KOCKER M, MAAROUF M, BENDEL M, VOGES J, MULLER RP, STURM V: Linac radiosurgery versus whole brain radiotherapy for brain metastases. A survival comparison based on the RTOG recursive partitioning analysis. Strahlenther Onkol 180:262-7, 2004.

29. KONDZIOLKA D, LUNSFORD D, WITT TC, FLICKINGER JC: The future of radiosurgery: radiobiology, technology, and applications. Surg Neurol 54:406-14, 2000.

30. KONDZIOLKA D, NIRANJAN A, FLICKINGER JC, LUNSFORD LD: Radiosurgery with or without whole-brain radiotherapy for brain metastases: the patients' perspective regarding complications. Am J Clin Oncol 28:173-9, 2005.

31. LI B, YU J, SUNTHARALINGAM M, KENNEDY AS, AMIN PP, CHEN Z et al.: Comparison of three treatment options for single brain metastasis from lung cancer. Int $\mathrm{J}$ Cancer (Radiat Oncol Invest) 90:37-45, 2000.

32. LIPPITZ BE, KRAEPELIEN T, HAUTANEN K, RITZLING M, RAHN T, ULFARSSON E et al.: Gamma knife radiosurgery for patients with multiple cerebral metastases. Acta Neurochir (Suppl) (Wien) 91:79-87, 2004.

33. LIU Y, XIAO S, LIU M, WANG D, HE J, HU B et al.: Analysis of related factors in complications of stereotactic radiosurgery in intracranial tumors. Stereotact Funct Neurosurg 75:129-32, 2000.

34. LOEFFLER JS, SHRIVE DC: What is appropriate therapy for patients single brain metastases? Int J Radiation Oncology Biol Phys 29:915-7, 1994.

35. MATSUO T, SHIBATAS, YASUNAGAA, IWANAGAM, MORI $\mathrm{K}, \mathrm{SHIMIZU} T$ et al.: Dose optimization and indication of linac radiosurgery for brain metastases. Int J Radiation Oncology Biol Phys 45: 931-9, 1999.

36. MEHTA M, NOYES W, LAMOND J, AUCHTER R, FRENCH M, JOHNSON $M$ et al.: A cost-effectiveness and cost-utility analysis of radiosurgery vs. resection for single-brain metastases. Int J Radiation Oncology Biol Phys 39:445-54, 1997.

37. MELVER JI, POLLOCK BE: Radiation-induced tumor after stereotactic radiosurgery and whole brain radiotherapy: case report and literature review. J Neurooncol 66:301-5, 2004.

38. MUACEVIC A, KRETH FW, MACK A, TONN JC, WOWRA $B$ : Stereotactic radiosurgery without radiation therapy providing high local control of multiple brain metastases from renal cell carcinoma. Minim Invasive Neurosurg 47:203-8, 2004.

39. MUACEVIC A, KRETH FW, TONN JC, WOWRA B: Stereotactic radiosurgery for multiple brain metastases from breast carcinoma. Cancer 100:1705-11, 2004.

40. NEIDER C, GROSU AL, GRZADZIEL A, SCHLEGEL J, MOLLS M: Brain metastases in renal cell cancer: diagnostic and therapeutic aspects. Am J Clin Oncol 27:632-4, 2004. 
41. NOEL G, SIMON JM, VALERY CA, CORNU P, BOISSERIE G, HASBOUN D et al.: Radiosurgery for brain metastases: impact of CTV on local control. Radiother Oncol 68:15-21, 2003.

42. NOEL G, SIMON JM, VALERY CA, CORNU P, BOISSERIE G, LEDU D et al.: Linac radiosurgery for brain metastases of melanoma. Stereotact Funct Neurosurg 79:245-55, 2002

43. NOEL G, VALERY CA, BOISSERIE G, CORNU P, HASBOUN $D$, SIMON MJ et al.: LINAC radiosurgery for brain metastasis of renal cell carcinoma. Urol Oncol 22:25-31, 2004.

44. O'NEILL BP, ITURRIA NJ, LINK MJ, POLLOCK BE, BALLMAN KV, O'FALLON JR: A comparison of surgical resection and stereotactic radiosurgery in the treatment of solitary brain metastases. Int J Radiat Oncol Biol Phys 55:1169-76, 2003.

45. OKUNIEFF P, SCHELL MC, RUO R, HALE ER, O'DELL WG, PILCHER W: Long-term management of patients with multiple brain metastases after shaped beam radiosurgery. Case report and review of the literature. J Neurosurg 101(Suppl 3):406-12, 2004.

46. PAN HC, SHEEHAN J, STROILAM, STEINER M, STEINER L: Gamma knife surgery for brain metastases from lung cancer. J Neurosurgery 102:28-33, 2005.

47. PATCHELL RA: A randomized trial of surgery in the management of single metastases to the brain. $\mathrm{N}$ Engl J Med 322:494-500, 1990

48. PAYNE BR, PRASAD D, SZEIFERT G, STEINER M, STEINER L: Gamma surgery for intracranial metastases from renal cell carcinoma. J Neurosurg 92:760-5, 2000.

49. PETROVICH Z, YU C, GIANNOTTA SL, O'DAY S, APUZZO $M L$ : Survival and pattern of failure in brain metastases treated with stereotactic gamma knife radiosurgery. J Neurosurg 97(5 Suppl):499-506, 2002.

50. RADBILL AE, FIVEASH JF, FALKENBERG ET, GUTHRIE BL, YOUNG PE, MELETH $S$ et al.: Initial treatment of melanoma brain metastases using gamma knife radiosurgery: an evaluation of efficacy and toxicity. Cancer 101: 825-33, 2004

51. REGINE WF, SCHMITT FA, SCOTT CB, DEARTH C, PATCHELL RA, NICHOLS RC Jr et al.: Feasibility of neurocognitive outcome evaluations in patients with brain metastases in a multi-institutional cooperative group setting: results of Radiation Therapy Oncology Group trial BR-0018. Int J Radiat Oncol Biol Phys 58:1346-52, 2004.

52. SANSUR CA, CHIN LS, AMES JW, BANEGURA AT, AGGARWAL S, BALLESTEROS $C$ et al.: Gamma knife radiosurgery for the treatment of brain metastases. Stereotact Funct Neurosurg, 74:37-51, 2000.

53. SCHOGGL A, KITZ K, REDDY M, WOLFSBERGER S, SCHNEIDER B, DIECKMANN $K$ et al.: Defining the role of stereotactic radiosurgery versus microsurgery in the treatment of single brain metastases. Acta Neurochir (Wien) 142:621-6, 2000.

54. SCHOMAS DA, ROESKE JC, MACDONALD RL, SWEENEY PJ, MEHTAN, MUNDTAJ: Predictors of tumor control in patients treated with linac-based stereotactic radiosurgery for metastatic disease to the brain. Am J Clin Oncol 28:180-7, 2005.

55. SHAW E, SCOTT C, SOUHAMI L, DINAPOLI R, KLINE R, LOEFFLER $\mathrm{J}$ et al.: Single dose radiosurgical treatment of recurrent previously treated primary brain tumors and metastases: final report of RTOG PROTOCOL 90-05. Int J Radiation Oncology Biol Phys 47:291-8, 2000.
56. SHEEHAN JP, SUN MH, KONDZIOLKA D, FLICKENGER J, LUNSFORD LD: Radiosurgery in patients with renal cell carcinoma metastasis to the brain: long-term outcomes and prognostic factors influencing survival and local tumor control. J Neurosurg 98:342-9, 2003.

57. SHEEHAN JP, SUN MH, KONDZIOLKA D, FLICKINGER J, LUNSFORD LD: Radiosurgery for non-small cell lung carcinoma metastatic to the brain: long-term outcomes and prognostic factors influencing patient survival time and local tumor control. J Neurosurg 97:1276-81, 2002.

58. SHEHATA MK, YOUNG B, REID B, PATCHELL RA, ST CLAIR W, SIMS J et al.: Sterotactic radiosurgery of 468 brain metastases $\leq 2 \mathrm{~cm}$ : implications for SRS dose and whole brain radiation therapy. Int J Radiat Oncol Biol Phys 59:87-93, 2004.

59. SHINOURA N, YAMADA R, OKAMOTO K, NAKAMURA O, SHITARA N: Local recurrence of metastatic brain tumor after stereotactic radiosurgery or surgery plus radiation. J Neurooncol 60:71-7, 2002.

60. SHUTO T, FUJINO H, ASADA H, INOMORI S, NAGANO $\mathrm{H}$ : Gamma knife radiosurgery for metastatic tumours in the brain stem. Acta Neurochir (Wien) 145:755-60, 2003.

61. SIMONOVA G, LISCAK R, NOVOTNY J, NOVOTNY J: Solitary brain metastases treated with the Leksell gamma knife: prognostic factors for patients. Radiother Oncol 57:207-13, 2000

62. SIMS E, DOUGHTY E, MACAULAY E, ROYLE N, WRAITH $C$, DARLISON $R$ et al.: Stereotactally delivered cranial radiation therapy: A ten year experienced of Linac based radiosurgery in the UK. Clin Oncol 11:303-20, 1999.

63. SPERDUTO PW: A review of stereotactic radiosrgery in the management of brain metastases. Technol Cancer Res Treat 2:105-10, 2003

64. STURM V, KOBER B, HOVER KH: Stereotactic percutaneous single dose irradiation of brain metastases with linear accelerator. Int $\mathrm{J}$ Radiation Oncol Biol Phys 13:279-82, 1987.

65. SUZUKI H, TOYODA S, MURAMATSU M, SHIMIZU T, KOJIMA T, TAKI W: Spontaneous haemorrhage into metastatic brain tumors after stereotactic radiosurgery using a linear accelerator. J Neurol Neurosurg Psychiatry 74: 908-12, 2003.

66. SUZUKI S, OMAGARI J, NISHIO S, NISHIYE E, FUKUI M: Gamma knife radiosurgery for simultaneous multiple brain metastatic brain tumors. J Neurosurg (Suppl 3) 93:30-31, 2000.

67. SZEIFERT GT, MASSAGER N, DEVRIENDT D, DAVID P, DE SMEDT F, RORIVE S et al.: Observations of intracranial neoplasm treated with gamma knife radiosurgery. J Neurosurg 97(5 Suppl):623-6, 2002.

68. SZEIFERT GT, SALMON I, RORIVE S, MASSAGER N, DEVRIENDT D, SIMON $S$ et al.: Does gamma knife surgery stimulate cellular immune response to metastatic brain tumors? A histopathological and immunohistochemical study. J Neurosurg 102 (Suppl):180-4, 2005.

69. TAKAHASHI M, NARABAYASHI I, KUROIWAT, UESUGI Y, TATSUMI T, INOMATA T: Stereotactic radiosurgery (SRS) for multiple metastatic brain tumors: effects of the number of target tumors on exposure dose in normal brain tissues. Int J Clin Oncol 8:289-96, 2003.

70. TARHINIAA,AGARWALASS: Management of brain metástases in patients with melanoma. Curr Opin Oncol 16:161-6, 2004.

71. ULM AJ, FRIEDMAN WA, BOVA FJ, BRADSHAW P, AMDUR RJ, MENDENHALL WM: Linear accelerator radiosur- 
gery in the treatment of brain metastases. Neurosurgery 55:1076-85, 2004.

72. VAN DER KOEGEL A: Central nervous system radiation injury in animals models. In GUTIN P, LEIBEL S, SHELINE $G$ (eds): Radiation injury to the nervous system. New York, Raven Press, 1991, pp 91-111.

73. WELLIS G, NAGEL R, VOLLMAR C, STEIGER HJ: Direct costs of microsurgical management of radiosurgically amenable intracranial pathology in Germany: an analysis of meningeomas, acoustic neuromas, metastases and arteriovenous malformations of less than $3 \mathrm{~cm}$ in diameter. Acta Neurochir (Wien) 145:249-55, 2003.

74. WELTMAN E, SALVAJOLI JV, BRANDT RA, HANRIOT RM, PRISCO FE, CRUZ JC et al.: Radiosurgery for brain metastases: a score index for predicting prognosis. Int $\mathrm{J}$ Radiation Oncol Biol Phys 46: 1155-61, 2000.

75. WOWRA B, MUACEVIC A, JESS-HEMPEN A, TONN JC: Safety and efficacy of outpatient gamma knife radiosurgery for multiple cerebral metastases. Expert Rev Neurother 4:673-9, 2004.

76. YU JS, YOUNG WH, WILSON D, BLACK KL: Glioblastoma induction after radiosurgery for meningeoma. Lancet 356:1576-7, 2000.

Original recebido em fevereiro de 2006

Aceito para publicação em abril de 2006

\section{Endereço para correspondência:}

Marcos Vinícius Calfat Maldaun

Rua Barata Ribeiro, 414 - cj. 63

01308-000 - São Paulo, SP

E-mail: marcosmaldaun@hotmail.com 